\title{
Impact of chemotherapy on eosinophilia-associated advanced rectal cancer: A case report and review of the literature
}

\author{
MAKI INOUE $^{1}$, JUN KADONO $^{1}$, HIROSHI SUGITA $^{2}$, TOSHIHIRO NAKAZONO $^{1}$, \\ SHUNSUKE MOTOI $^{1}$, IWAO KITAZONO ${ }^{1}$, YUKO GOTO ${ }^{3}$, YOSHIHIKO FUKUKURA ${ }^{4}$, \\ MAKOTO YOSHIMITSU ${ }^{5}$, TAKAHARU MISAKA ${ }^{2}$ and YUTAKA IMOTO ${ }^{1}$ \\ ${ }^{1}$ Department of Cardiovascular and Gastroenterological Surgery, \\ Kagoshima University Medical and Dental Hospital, Kagoshima 890-8544; ${ }^{2}$ Department of Internal Medicine, \\ Kirishima Medical Center, Kirishima, Kagoshima 899-5112; Departments of ${ }^{3}$ Human Pathology and ${ }^{4}$ Radiology; \\ ${ }^{5}$ Division of Hematology and Immunology, Kagoshima University Medical and Dental Hospital, Kagoshima 890-8544, Japan
}

Received March 27, 2015; Accepted April 15, 2016

DOI: 10.3892/ol.2016.5364

\begin{abstract}
The present study reports a case of eosinophilia-associated rectal cancer that was successfully stabilized using chemotherapy, and reviews the mechanisms of eosinophilia and the importance of chemotherapy. A 65-year-old man, who had previously been diagnosed with suspected rectal cancer, presented with the chief complaint of melena. Eosinophilia, abnormal blood coagulation, and elevated carcinoembryonic antigen and carbohydrate antigen 19-9 tumor marker levels were observed, and the patient was subsequently diagnosed with advanced rectal cancer accompanied by multiple lymph node metastases that extended from the para-aortic lymph nodes to the left axillary lymph nodes. The complication of deep vein thrombosis was also observed. Tumor hemorrhage was exacerbated, and thus, Hartmann's procedure was performed. Pathological findings included poorly- to moderately-differentiated adenocarcinoma; however, no eosinophil infiltration was observed within the tumor. Following surgery, the eosinophilia and lymph node metastasis were exacerbated, and an oxaliplatin plus capecitabine chemotherapy regimen was initiated. The patient's eosinophil count and tumor marker levels normalized, and the lymph nodes decreased in size; however, re-enlargement of the lymph nodes was observed 6 months after surgery. The
\end{abstract}

Correspondence to: Dr Maki Inoue, Department of Cardiovascular and Gastroenterological Surgery, Kagoshima University Medical and Dental Hospital, 8-35-1 Sakuragaoka, Kagoshima 890-8544, Japan

E-mail: inoue@m.kufm.kagoshima-u.ac.jp

Abbreviations: IL, interleukin; CT, computed tomography; XELOX, oxaliplatin plus capecitabine; FOLFIRI, irinotecan/fluorouracil/folinic acid; Bev, bevacizumab; DIC, disseminated intravascular coagulopathy

Key words: rectal cancer, eosinophilia, paraneoplastic syndrome, chemotherapy, deep venous thrombosis patient was then administered a chemotherapeutic regimen of irinotecan/fluorouracil/folinic acid + bevacizumab, and stable disease was maintained until pleural and peritoneal dissemination were observed at 22 months post-surgery. Following a rapid deterioration in condition, the patient succumbed to the disease at 23 months post-surgery. The present case indicates that although eosinophilia-associated colon cancer exhibits a poor prognosis, early chemotherapeutic intervention may improve this.

\section{Introduction}

Eosinophilia is known to cause complications in allergic disease and parasitic infections, and may occasionally cause complications in malignant tumors. Previous studies have indicated that eosinophilia-associated complications may arise not only in hematopoietic malignancies, but also in lung cancer (1), gastric cancer (2) and renal cell carcinoma (3); however, the mechanism has not been elucidated. Approximately $0.5-1.0 \%$ of cases of eosinophilia are associated with malignancies (2-4). Eosinophilia in solid malignancies is rarely reported, and there are a limited number of cases describing eosinophilia in rectal cancer. A number of previously reported cases have involved advanced cancers accompanied by metastases, and the majority have exhibited a poor prognosis. Peripheral hypereosinophilia is considered to be a poor prognostic sign, which is frequently associated with extensive metastatic disease (4-8) The present study reports the case of a patient with eosinophilia-associated advanced rectal cancer, which was successfully stabilized using chemotherapy. The possible mechanism underlying eosinophilia-associated complications in the present case and the importance of early chemotherapeutic intervention in treating such cancers are also reviewed.

\section{Case report}

In February 2013, a 65-year-old man presented to the Kirishima Medical Center (Kirishima, Japan) with the primary complaint of melena, and was diagnosed with rectal 
cancer. The patient had tested positive for fecal occult blood during a routine medical examination 1 year earlier, but had left the condition uncontrolled. The patient's prior medical history included an unspecified joint surgery to the left shoulder 6 years earlier. During a visit to the Department of Cardiovascular and Gastroenterological Surgery, Kagoshima University Medical and Dental Hospital (Kagoshima, Japan) in March 2013, the patient presented with severe anemia caused by tumor hemorrhage and was immediately hospitalized with a diagnosis of hemorrhagic rectal cancer. Upon hospital admission, the baseline characteristics were as follows: Body temperature, $36.6^{\circ} \mathrm{C}$ (normal range, $36.6-37.0^{\circ} \mathrm{C}$ ); blood pressure, $105 / 60 \mathrm{mmHg}$ (normal range, $<120 / 80 \mathrm{mmHg}$ ); and pulse rate, 83 beats/min (normal range, $50-80$ beats $/ \mathrm{min}$ ). Physical examination revealed swelling of the left upper extremity. In addition, the abdomen was flat, soft and non-tender, and no tumor was palpable. Digital rectal examination identified a mass accompanied by bleeding proximal to the left anterior wall, located $\sim 7 \mathrm{~cm}$ from the anal verge. Laboratory blood test results upon admission were as follows: White blood cells, 15,630/ $\mu 1$ (normal range, 4,500-8,500/ $\mu 1$ ); eosinophils, 39\% (normal range, 1-4\%); hemoglobin, $6.1 \mathrm{~g} / \mathrm{dl}$ (normal range, $14.0-18.0 \mathrm{~g} / \mathrm{dl}$ ); platelets, $11.1 \times 10^{4} / \mu 1$ (normal range, $13.0-32.0 \times 10^{4} / \mu 1$ ); prothrombin time-international normalized ratio, 2.17 (normal range, 0.85-1.15); and activated partial thromboplastin time, $57.7 \mathrm{sec}$ (normal range, 25.1-36.5 sec). These results indicated coagulopathy. Furthermore, the lactate dehydrogenase level was mildly increased to $288 \mathrm{IU} / 1$ (normal range, 119-229 IU/1). Hepatorenal function tests revealed normal results as follows: Aspartate aminotransferase, $16 \mathrm{IU} / 1$ (normal range, 13-33 IU/l); alanine aminotransferase, $13 \mathrm{IU} / 1$ (normal range, 6-30 IU/l); urea nitrogen, $19.4 \mathrm{mg} / \mathrm{dl}$ (normal range, $8-22 \mathrm{mg} / \mathrm{dl}$ ); and creatinine, $0.82 \mathrm{mg} / \mathrm{dl}$ (normal range, $0.6-1.1 \mathrm{mg} / \mathrm{dl}$ ).

Elevated levels of tumor markers were observed as follows: Carcinoembryonic antigen, $9.1 \mathrm{ng} / \mathrm{ml}$ (normal range, $<5.68 \mathrm{ng} / \mathrm{ml}$ ) and carbohydrate antigen $19-9,72.6 \mathrm{U} / \mathrm{ml}$ (normal range, $<37.0 \mathrm{U} / \mathrm{ml}$ ). A stool examination for parasites and ova yielded negative results. Lower gastrointestinal endoscopy revealed a full-circumference tumor accompanied by bleeding in the mid-rectum, which obstructed the passage of the endoscope probe. Chest/abdominal computed tomography $(\mathrm{CT})$ revealed irregular wall thickening extending from the sigmoid colon to the upper rectum, and extraserosal invasion of the rectal tumor was suspected (Fig. 1A). Enlarged para-aortic, supraclavicular and left axillary lymph nodes were observed, in addition to swelling of the regional lymph nodes. A thrombus was also observed in the subclavian/brachiocephalic vein, extending from the left internal jugular vein (Fig. 1B). No liver or lung metastasis was observed, and no peritoneal dissemination was identified. Positron emission tomography/CT revealed fluorodeoxyglucose accumulation in the rectum and lymph nodes, in the region where swelling was indicated on the CT scans. The maximum standardized uptake values were 15.8 and 9-14.1 in the rectum and lymph nodes, respectively (Fig. 2). Based on the aforementioned observations, malignant lymphoma was considered as a differential diagnosis; however, the lactate dehydrogenase level was mildly elevated in relation to the tumor size, and as no other organ was involved, the patient
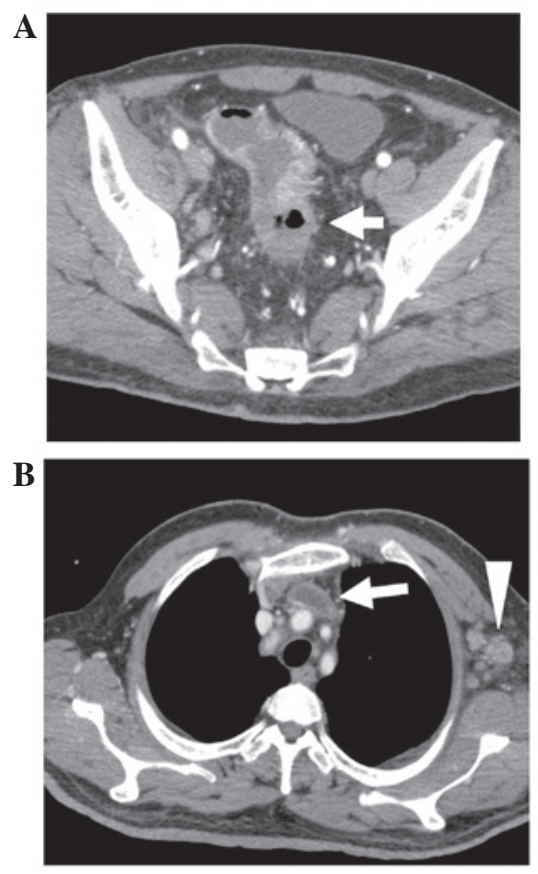

Figure 1. (A) Computed tomography on admission showing irregular rectal wall thickness (arrow), (B) swelling in the left axillary lymph nodes (arrowhead) and a thrombus in the brachiocephalic vein (arrow).

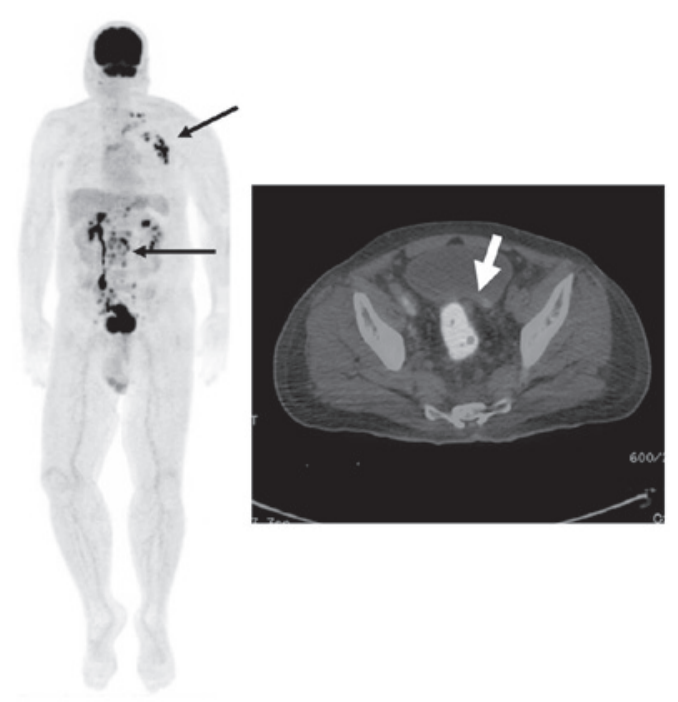

Figure 2. Positron emission tomography/computed tomography showing fluorodeoxyglucose uptake in the rectum and multiple lymph nodes (arrows).

was diagnosed with T4aN2bM1a, stage IVA rectal cancer, according to the International Union Against Cancer (9). Concentrated red blood cells $(1,680 \mathrm{ml})$ and fresh frozen plasma $(960 \mathrm{ml})$ were administered; however, the melena persisted and the anemia progressed. Hematuria was also identified. Subsequently, the patient's condition deteriorated due disseminated intravascular coagulation (DIC). Therefore, a primary tumor resection was performed immediately to control the bleeding. A laparotomy surgical procedure was performed with an incision in the lower abdominal midline. No peritoneal dissemination was observed. The tumor of the rectosigmoid colon had directly infiltrated the bladder via the 


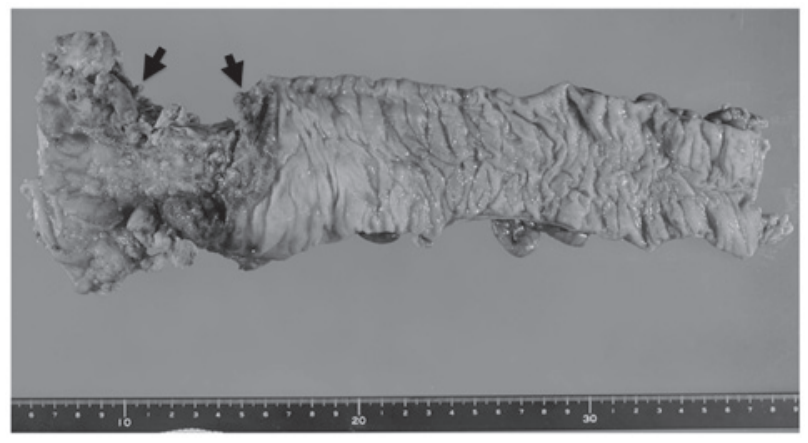

Figure 3. Resected rectal cancer showing a circumferential type 2 tumor (arrows).
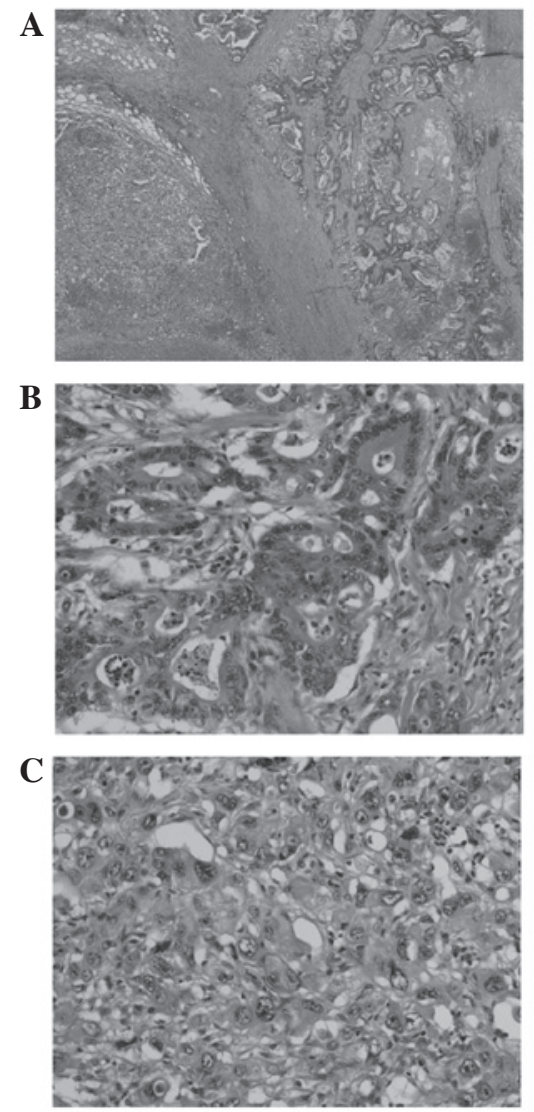

Figure 4. (A) Histopathological examination of the rectum showing poorly- to moderately-differentiated adenocarcinoma (hematoxylin and eosin stain; magnification, $\mathrm{x} 20$ ). Negligible eosinophil infiltration is visible in the (B) moderately- and (C) poorly-differentiated adenocarcinoma (hematoxylin and eosin stain; magnification, $\mathrm{x} 200$ ).

upper rectum. As a non-curative resection was performed, concomitant resection of the bladder was not achieved. Therefore, the infiltrated portion of the bladder was detached and Hartmann's procedure was performed. Hemostasis was difficult to achieve inside the pelvic cavity, however, it was obtained using compression, hemostatic agents (absorbable hemostat and fibrin adhesive; Surgicel NU-KNIT; Ethicon, Somerville, NJ, USA) and a blood transfusion (red blood cells, 1,920 ml; fresh frozen plasma, $800 \mathrm{ml}$ ).

The resected specimen revealed a circumferential type 2 tumor, based on the Japanese Classification of

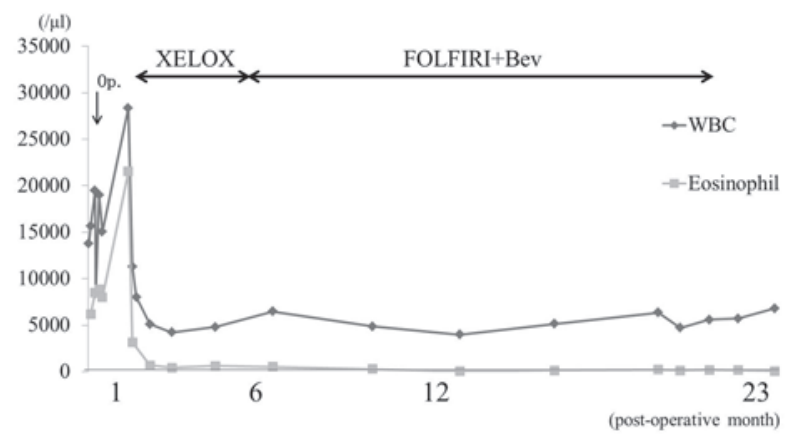

Figure 5. Routine post-operative measurements of WBC and eosinophil levels. XELOX, oxaliplatin plus capecitabine; FOLFIRI + Bev, irinotecan/5-fluorouracil/folinic acid + bevacizumab; WBC, white blood cell.
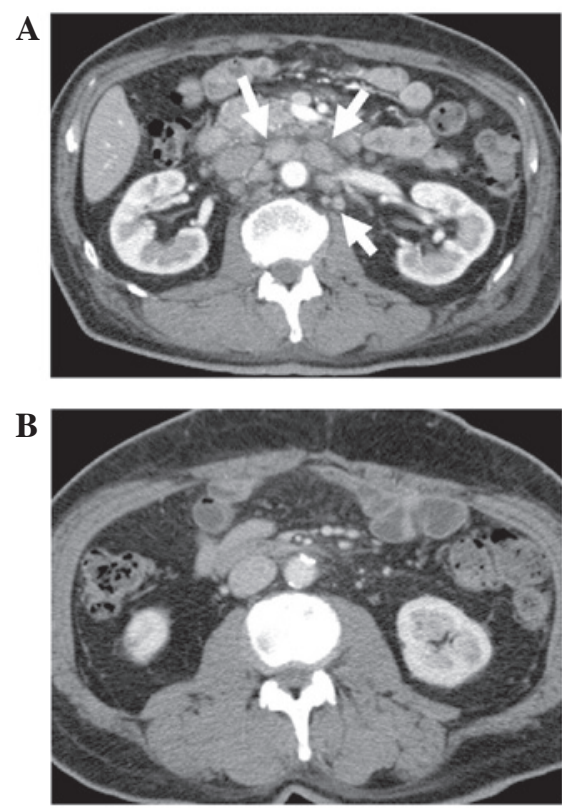

Figure 6. Computed tomography showing metastases in the para-aortic lymph nodes (arrows) (A) prior to the induction of chemotherapy and (B) following 3 courses of oxaliplatin plus capecitabine therapy.

Colorectal Carcinoma (8th edition) (10), 95x50 $\mathrm{mm}$ in size (Fig. 3). Following histopathological examination, the patient was diagnosed with an admixture of poorly- and moderately-differentiated adenocarcinoma, accompanied by advanced venous-lymphatic infiltration (Fig. 4A). Histological examination revealed a mixture of mucinous adenocarcinoma, with significant mucus production at the periphery of the tumor cell nest. Metastasis was observed in 12/19 of the dissected lymph nodes. Minimal eosinophil infiltration into the tumor tissue was observed (Fig. 4B and C). Metastases of the inferior mesenteric lymph nodes were predominantly composed of moderately-differentiated adenocarcinoma cells.

Following surgery, the eosinophil count in the peripheral blood was immediately reduced, however, by post-operative day 21 , it had increased to $21,000 / \mu 1$. Interleukin (IL)-2 receptor levels were mildly elevated $(1,464 \mathrm{U} / \mathrm{ml}$; normal range, 127-582 U/ml)), bone marrow examination did not reveal any leukemic blasts and fluorescence in situ hybridization analysis, for the identification of the factor interacting with 
Table I. Previously reported cases of colorectal cancer with eosinophilia.

\begin{tabular}{|c|c|c|c|c|c|c|c|}
\hline First author, year & $\begin{array}{l}\text { Age, } \\
\text { years }\end{array}$ & Gender & $\begin{array}{l}\text { Tumor } \\
\text { localization }\end{array}$ & Histological type & $\begin{array}{l}\text { Tumor } \\
\text { stage }\end{array}$ & Outcome & (Ref.) \\
\hline Anagnostopoulos et al, 2005 & 47 & $\mathrm{~F}$ & Cecum & tub2 & IV & $\begin{array}{l}\text { DOD at } 12 \text { months } \\
\text { post-diagnosis }\end{array}$ & (4) \\
\hline Tajima et al, 1998 & 76 & $\mathrm{~F}$ & Rectum & tub2 & III & NR & (13) \\
\hline Uemura et al, 2004 & 33 & M & $\begin{array}{l}\text { Ascending, } \\
\text { transverse }\end{array}$ & NR & NR & $\begin{array}{l}\text { DOD at } 27 \text { days } \\
\text { post-diagnosis, } \\
\text { pulmonary emboli }\end{array}$ & (18) \\
\hline Kato et al, 2010 & 72 & F & Ascending & por & IV & $\begin{array}{l}\text { DIC, DOD at } 1 \text { month } \\
\text { post-diagnosis }\end{array}$ & (19) \\
\hline Present study & 65 & M & Rectum & por + tub $2+$ muc & IV & $\begin{array}{l}\text { DOD at } 23 \text { months } \\
\text { post-diagnosis }\end{array}$ & \\
\hline
\end{tabular}

F, female; M, male; tub2, moderately-differenitiated adenocarcinoma; muc, mucinous adenocarcinoma; por, poorly-differentiated adenocarcinoma; DOD, died of disease; NR, not reported; XELOX, oxaliplatin plus capecitabine; FOLFIRI, irinotecan/fluorouracil/folinic acid; DIC, disseminated intravascular coagulopathy.

PAPOLA and CPSF1-platelet derived growth factor receptor $\alpha$ (FIP1L1-PDGFRA) fusion gene, revealed no chromosomal abnormalities. Therefore, the possibility of chronic eosinophilic leukemia was excluded. Blood concentrations of granulocyte macrophage colony-stimulating factor, IL-3 and IL-5 were also measured, and were all within the reference values. The initiation of chemotherapy was delayed by 1 month due to the complication of a deep surgical site infection. On post-operative day 32 , the patient was administered oxaliplatin $\left(130 \mathrm{mg} / \mathrm{m}^{2}\right.$, day 1$)$ plus capecitabine $(1,000 \mathrm{mg}$, twice daily, days 1-14) (XELOX) therapy. Following 13 -week cycle of chemotherapy, the eosinophil count decreased to 666/ $\mu 1$ (Fig. 5). After the completion of 3 cycles of XELOX therapy, the lymph nodes, including the para-aortic lymph nodes, exhibited a marked reduction in size (Fig. 6), and the carcinoembryonic antigen and carbohydrate antigen 19-9 the tumor marker levels normalized to $4.1 \mathrm{ng} / \mathrm{ml}$ and $36.7 \mathrm{U} / \mathrm{ml}$, respectively. However, following the completion of 6 cycles of XELOX therapy, enlargement of the iliac lymph nodes was observed. Subsequently, the chemotherapy regimen was changed to the following: FOLFIRI [Leucovorin $200 \mathrm{mg} / \mathrm{m}^{2}$ intravenous (i.v.) prior to fluorouracil (5-FU) on day 1 and 5 -FU $500 \mathrm{mg} / \mathrm{m}^{2}$ i.v. bolus on day 1 ; then $2,400 \mathrm{mg} / \mathrm{m}^{2}$ i.v. over $46 \mathrm{~h}$ and irinotecan $180 \mathrm{mg} / \mathrm{m}^{2}$ i.v. over $90 \mathrm{~min}$ on day 1; in a 2-week cycle) plus Bev (5 mg/kg i.v. bolus on day 1$)$. A total of 29 cycles of chemotherapy (FOLFIRI+Bev) were administered. Stable disease was then maintained until peritoneal and pleural dissemination were observed at 22 months post-surgery. The patient's condition deteriorated rapidly and multiple skin nodules developed, which were composed primarily of poorly-differentiated adenocarcinoma. The patient succumbed to the disease at 23 months post-surgery.

\section{Discussion}

Paraneoplastic syndromes are characterized by symptoms that are observed at sites distant from a primary tumor or metastases, occurring in $1-7.4 \%$ of tumor-bearing patients (11).
Paraneoplastic syndromes are clinically significant, as in certain cases they may present as the first symptoms of a cancer and thus, if patients consciously neglect to seek diagnosis and treatment, a diagnosis may not be reached and treatment may be delayed. Furthermore, the symptoms of paraneoplastic syndromes may worsen in association with the progression of the tumors (12). Eosinophilia, which is considered a component of paraneoplastic syndromes, has been reported not only in hematopoietic malignancies, but also in solid tumors $(1-3,5,13)$. In addition, it has been hypothesized that IL-3, IL-5 and other cytokines are involved in the differentiation and maturation of eosinophils $(1,5,13,14)$. An association between bone marrow carcinogenesis and eosinophilia has been suggested (15), and although it is rare, previous studies have reported cases of bone marrow carcinogenesis accompanied by colon cancer $(15,16)$. The patient in the present case underwent a blood transfusion to control bleeding caused by tumor hemorrhage and DIC, and then underwent emergency surgery; therefore, it was not possible to measure cytokine levels or perform bone marrow tests prior to surgery. However, the levels of IL-3, IL-5 and granulocyte-macrophage colony-stimulating factor in the blood were all within the normal ranges when measured prior to the initiation of chemotherapy, when the residual tumor was highly active. Bone marrow examination did not identify leukemic blasts and thus, the possibility of bone marrow carcinogenesis was excluded. The possibility of a myeloid tumor accompanied by a genetic abnormality was also considered, however, an examination to identify the FIP1L1-PDGFRA fusion gene (17) did not identify any chromosomal abnormalities. Therefore, the specific factors involved in the eosinophilia were not identified in the present case and the mechanism remains unknown. However, as the eosinophil count was strongly associated with tumor activity, we hypothesize that the tumor itself produced the factors required for the development of eosinophilia.

In the present case, the administration of coagulation factors to control venous thrombosis exacerbated the patient's 
tendency to bleed, making it difficult to control the bleeding from the tumor. The presence of a malignant tumor has been considered to contribute to a thrombotic tendency, whereby eosinophils produce granular proteins, such as the eosinophil cationic protein, and induce vascular endothelial dysfunction and inactivation of thrombomodulin, in order to enhance coagulation. Therefore, patients with eosinophilia may experience severe thrombosis complications $(2,18)$. Furthermore, in the present case, the possibility of adverse effects from the previous shoulder joint surgery cannot be ignored as one of the causes; however, as a long time had elapsed since the surgery, and rapid improvement of the edema in the upper left extremity was observed following anti-coagulation therapy and chemotherapy, we hypothesize that a novel thrombus, formed as a result of eosinophilia and the tumor, rather than a chronic thrombus, was involved

Reports of colon cancer accompanied by eosinophilia are rare. Upon conducting a literature review of studies published between 1946 and 2014 using PubMed, using the keywords 'eosinophilia' and 'colon cancer', only 5 cases were identified, including the present case $(4,13,18,19)$ (Table I). The 4 previous studies included poorly-differentiated adenocarcinomas, which were highly malignant in 2 cases, including the present case. Distant metastasis or disseminated disease are often observed at the time of diagnosis and thus, patients may succumb due to DIC or embolism prior to the initiation of chemotherapy, therefore the prognosis is extremely poor $(4,18,19)$. In the present case, XELOX therapy significantly reduced the size of the lymph node metastases, and the tumor marker levels and eosinophil counts also normalized. The patient was administered FOLFIRI + Bev for the exacerbated lesions 6 months later and a stable disease state was maintained for 15 months. Overall, tumor suppression was maintained for 21 months.

Due to the highly malignant nature of poorly-differentiated colon cancer, the mean post-operative survival time is 8.3 months, representing a poor prognosis (20), and to date, no standard chemotherapy regimen has been established. In the present case, equal proportions of moderately- and poorly-differentiated adenocarcinomas were observed within the primary lesion, whereas the lymph node metastases were primarily composed of moderately-differentiated adenocarcinoma. No histopathological analysis of the distant metastases was performed and thus, no conclusions could be drawn; however, it is possible that the chemotherapy was successful and contributed to improving the prognosis, as the distant metastases were primarily composed of the moderately-differentiated type. Among the previously reported cases, the only patient with moderately-differentiated adenocarcinoma who underwent chemotherapy survived for 12 months (4). This indicates that although colon cancer accompanied by eosinophilia exhibits a poor prognosis, chemotherapy may be successful if the cancer is caused by a moderately-differentiated adenocarcinoma.

In the present case, after pleural-peritoneal dissemination developed, no enlargement of the lymph nodes or increase in eosinophil counts was observed. We hypothesize that eosinophilia was caused by the moderately-differentiated adenocarcinoma in the present case, based on the following observations: The skin metastasis was primarily composed of poorly-differentiated adenocarcinoma; the eosinophil count was normal despite the development of dissemination; the reduction in eosinophil count coincided with the chemotherapy-induced reduction of lymph node metastases; and the resected metastatic lymph nodes were primarily composed of moderately-differentiated adenocarcinoma. In the future, comprehensive analysis of the factors that contribute to cancer-associated eosinophilia, such as cytokines, is required.

In the present study, the administration of chemotherapy successfully stabilized eosinophilia-associated advanced rectal cancer following primary lesion resection. We hypothesize that moderately-differentiated adenocarcinoma was involved in the development of the eosinophilia. These observations indicate that, although eosinophilia-associated colon cancer exhibits a poor prognosis, early chemotherapy treatment may improve the prognosis if the tumor is primarily composed of moderately-differentiated adenocarcinoma cells.

\section{References}

1. Kundu S, Misra S, Haldar RK and Mitra R: Severe paraneoplastic peripheral blood eosinophilia and eosinophilic malignant pleural effusion as rare manifestation of squamous cell carcinoma of lung. Int Med J 20: 34-35, 2013.

2. Takeda H, Nishikawa H, Tsumura T, Sekikawa A, Maruo T, Okabe Y, Kimura T, Wakasa T and Osaki Y: Prominent hypereosinophilia with disseminated intravascular coagulation as an unusual presentation of advanced gastric cancer. Intern Med 53: 563-569, 2014

3. Wei YB, Yan B, Yin Z and Yang JR: Chromophobe renal cell carcinoma associated with eosinophilia: A report of three cases. Exp Ther Med 8: 91-94, 2014

4. Anagnostopoulos GK, Sakorafas GH, Kostopoulos P, Margantinis G, Tsiakos S, Terpos E, Pavlakis G, Fortun P and Arvanitidis D: Disseminated colon cancer with severe peripheral blood eosinophilia and elevated serum levels of interleukine-2, interleukine-3, interleukine-5 and GM-CSF. J Surg Oncol 89: 273-275, 2005.

5. Stefanini M, Claustro JC, Motos RA and Bendigo LL: Blood and bone marrow eosinophilia in malignant tumors. Role and nature of blood and tissue eosinophil colony-stimulation factor(s) in two patients. Cancer 68: 543-548, 1991.

6. Todenhöfer T, Wirths S, von Weyhern CH, Heckl S, Horger M, Hennenlotter J, Stenzl A, Kanz L and Schwentner C: Severe paraneoplastic hypereosinophilia in metastatic renal cell carcinoma. BMC Urol 12: 7, 2012

7. Chang WC, Liaw CC, Wang PN, Tsai YH and Hsueh S: Tumor-associated hypereosinophilia: Report of four cases. Changgeng Yi Xue Za Zhi 19: 66-70, 1996.

8. Lowe D, Jorizzo J and Hutt MS: Tumour-associated eosinophilia: A review. J Clin Pathol 34: 1343-1348, 1981.

9. Sobin LH, Gospodarowicz MK and Wittekind C (eds): TNM Classification of Malignant Tumours. 7th edition. Wiley-Blackwell, New York, NY, pp100-109, 2009.

10. Japanese Society for Cancer of the Colon and Rectum. Japanese Classification of Colorectal Carcinoma. 8th edition. Kanehara, Tokyo, 2013.

11. Baijens LW and Manni JJ: Paraneoplastic syndromes in patients with primary malignancies of the head and neck. Four cases and a review of the literature. Eur Arch Otorhinolaryngol 263: 32-36, 2006.

12. Pelosof LC and Gerber DE: Paraneoplastic syndromes: An approach to diagnosis and treatment. Mayo Clin Proc 85: $838-854,2010$

13. Tajima K, Yamakawa M, Inaba Y, Katagiri T and Sasaki H: Cellular localization of interleukin-5 expression in rectal carcinoma with eosinophilia. Hum Pathol 29: 1024-1028, 1998.

14. Fridlender ZG, Simon HU and Shalit M: Metastatic carcinoma presenting with concomitant eosinophilia and thromboembolism. Am J Med Sci 326: 98-101, 2003. 
15. Higashiyama A, Kudo M, Nagasako T, Kawamura N, Abiko S Yamamoto Y, Takano M, Gotoh J, Tamaki T, Meguro J, et al: Successful chemotherapy of carcinomatosis of the bone marrow with disseminated intravascular coagulation from a rectal carcinoma found by eosinophilia. Nihon Shokakibyo Gakkai Zasshi 108: 1244-1251, 2011 (In Japanese).

16. Naito M, Yoshida Y, Aisu N, Tanimura S, Hoshino S, Tanaka T, Nimura S, Tamura K and Yamashita Y: A report of disseminated carcinomatosis of the bone marrow originating from transverse colon cancer successfully treated with chemotherapy using XELOX plus bevacizumab. Case Rep Oncol 7: 426-434, 2014.

17. Cools J, Deangelo DJ, Gotlib J, Stover EH, Legare RD, Cortes J, Kutok J, Clark J, Galinsky I, Griffin JD, et al: A tyrosine kinase created by fusion of the PDGFRA and FIP1L1 genes as a therapeutic target of imatinib in idiopathic hypereosinophilic syndrome. N Engl J Med 348: 1201-1214, 2003.
18. Uemura K, Nakajim M, Yamauchi N, Fukayama M and Yoshida K: Sudden death of a patient with primary hypereosinophilia, colon tumours and pulmonary emboli. J Clin Pathol 57: $541-543,2004$.

19. Kato H, Kohata K, Yamamoto J, Ichikawa S, Watanabe M, Ishizawa K, Ichinohasama R and Harigae H: Extreme eosinophilia caused by interleukin-5-producing disseminated colon cancer. Int J Hematol 91: 328-330, 2010.

20. Kosuge M, Ogawa M, Watanabe M, Eto K, Yokoyama M and Yanaga K: A case of poorly differentiated adenocarcinoma of the transverse colon in which MTX/5-FU therapy was effective for disseminated intravascular coagulation syndrome due to carcinomatosis of bone marrow. J Jpn Surg Assoc 68: 943-947, 2007 (In Japanese). 